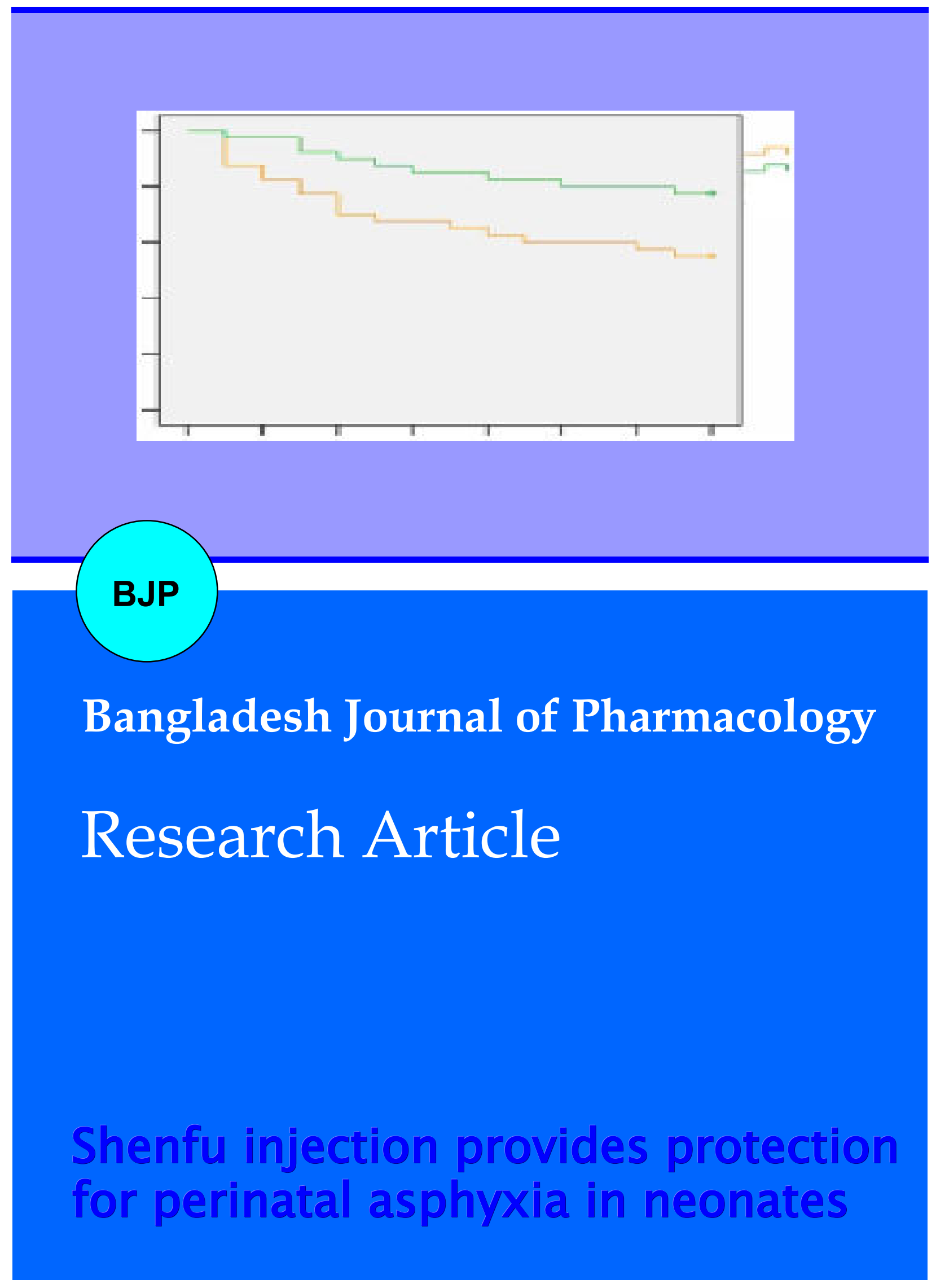


Abstracted/indexed in Academic Search Complete, Asia Journals Online, Bangladesh Journals Online, Biological Abstracts, BIOSIS Previews, CAB Abstracts, Current Abstracts, Directory of Open Access Journals, EMBASE/Excerpta Medica, Google Scholar, HINARI (WHO), International Pharmaceutical Abstracts, Open J-gate, Science Citation Index Expanded, SCOPUS and Social Sciences Citation Index;

ISSN: $1991-0088$

\title{
Shenfu injection provides protection for perinatal asphyxia in neonates
}

\author{
Yu Jiang1, Rong-Rong Zhang², Li-Juan Yang², Zhen $\mathrm{Wu}^{2}$, Huai-Ping Chen², Sai Zhao \\ and Zhao-Fang Tian'
}

${ }^{1}$ Department of Pediatrics, Huai'an Hospital Affiliated to Xuzhou Medical College and Huai'an Second People's Hospital, Huai'an, 223 300, China; ${ }^{2}$ Department of Neonatology, Huai'an First Hospital Affiliated to Nanjing Medical University, Huai'an, 223 300, China.

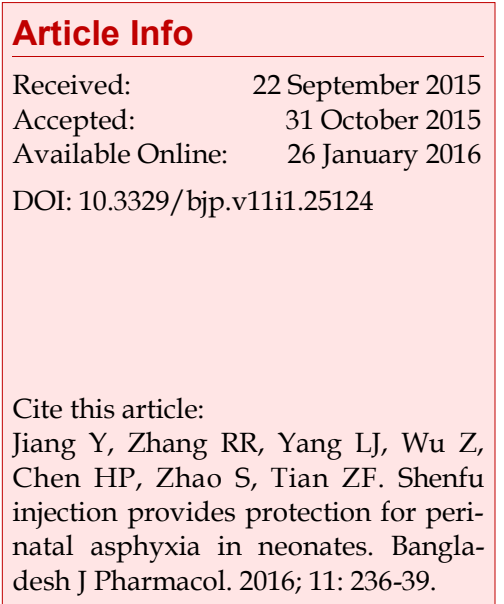

\begin{abstract}
This study aimed to investigate the efficacy of shenfu injection for the protection of neonates with asphyxia. Eighty neonates with asphyxia were randomly divided into two groups, treatment group and control group $(n=40)$. Both groups received interventions such as ventilation, oxygen, and circulation support. Treatment group was administrated with shenfu injection additionally. Serum levels of creatine kinase, alanine aminotransferase, aspartate aminotransferase, creatinine, and neuron-specific enolase were significantly lower but the oxygenation index was significantly higher in treatment group on day 7 and day 14. The neurobehavioral score was significantly higher in treatment group than in control group. On the $14^{\text {th }}$ day, the survival rate of treatment group (77.5\%) was higher than that of control group (55\%). Shenfu injection could protect the function of the brain, heart, lung, liver and kidney by attenuating ischemia reperfusion after severe asphyxia resuscitation, improve neurobehavioral ability and increase the survival of neonates.
\end{abstract}

\section{Introduction}

Perinatal asphyxia or birth asphyxia is a common disease in neonates and has very high rate of morbidity and mortality (Goel and Gupta, 2012). Perinatal asphyxia is mainly caused by the inadequate intake of the oxygen by the neonates before, during, or just after birth. Decreased oxygen intake leads to a series of biological and chemical changes in the body such as hypoxemia and acidosis. The management of perinatal asphyxia mainly focuses on providing supportive care to maintain the temperature, perfusion, ventilation and normal metabolic state including glucose, calcium and acid-base balance in neonates. Prompt and efficient neonatal resuscitation is an important approach for the treatment of perinatal asphyxia. However, hypoxia and hypoxemia before resuscitation, ischemia reperfusion after resuscitation, and stress responses in the body all account for multiple organ dysfunction such as the injuries to the heart, the liver, the kidney, the lung and the brain. Consequently, the survival rate of neonates after resuscitation is very low and even those who survive have a low quality of life (Leone and Finer, 2011).

Shenfu injection is made of the extracts from traditional Chinese herbs that mainly consist of ginsenoside Rb1 and aconitine alkaloids. Shenfu injection has shown protective effects on ischemia injuries to a variety of organs such as the heart, the liver, the kidney, the lung and the brain (Zheng et a1., 2015; Wang et al., 2008; Hou et a1., 2013; Ji et al., 2013; Guo and Li, 2013; Yin et al., 2014; Zhang et al., 2012). Therefore, in the present study we aimed to investigate the efficacy of shenfu injection for the protection of neonates after resuscitation treatment of asphyxia. 


\section{Materials and Methods}

\section{Patients and treatment}

Total 80 neonates with perinatal asphyxia were enrolled in this study who were admitted in our Department from June 2013 to May 2015. All neonates were diagnosed as perinatal asphyxia based on the symptoms and Apgar score. They were randomly divided into two equal number of two groups: Treatment group and control group. Both groups received routine interventions such as ventilation, oxygen, and circulation support, and the control of infection, convulsions, and intracranial pressure. In addition, treatment group was administrated with shenfu injection $(1 \mathrm{~mL} / \mathrm{kg}$, Ya'an 39 Pharmaceutical Co., Ltd.) for 7 days, while control was administrated with equal amount of saline.

Fasting peripheral venous blood was collected from each neonate on day 1 , day 7 and day 14 . Serum was separated and stored at $-80^{\circ} \mathrm{C}$ for subsequent analysis. Serum levels of creatine kinase, alanine aminotransferase, aspartate aminotransferase, creatinine, and neuron-specific enolase were detected by biochemical analyzer. In addition, oxygenation index $\left(\mathrm{PO}_{2} / \mathrm{FiO}_{2}\right)$ was evaluated on day 1 , day 7 and day 14 .

\section{Neonatal neurobehavioral test}

Neonatal neurobehavioral test was performed using 20item neonatal behavioral neurological assessment test as described previously (Bao et al., 1991). Each item has 3 scales $(0,1,2)$, and 20 items have a maximum of 40 scores. The higher scores indicated better neurobehavioral ability.

\section{Statistical analysis}

The data were expressed as mean \pm SD and analyzed using SPSS software (version 20.0). The comparison between groups was analyzed by t-test or $\chi^{2}$ test. The survival time was analyzed by Lograk test. $p<0.05$ was considered statistically significant.

\section{Results}

\section{Patient characteristics}

Control group included 21 males and 19 females, the age range was 37-42 weeks, the body weight range was $2.50-4.0 \mathrm{~kg}(3.4 \pm 0.5 \mathrm{~kg})$, Apgar score was 0-3 (2.0 \pm 0.4$)$ at $1 \mathrm{~min}$ after birth and $4-8(5.9 \pm 2.0)$ at 5 min after birth. Treatment group included 22 males and 18 females, the age range was 37-42 weeks, the body weight range was $2.50-4.0 \mathrm{~kg}(3.4 \pm 0.5 \mathrm{~kg})$, Apgar score was 0-3 (2.0 \pm 0.5$)$ at $1 \mathrm{~min}$ after birth and 4-8 (5.9 $\pm 1.9)$ at $5 \mathrm{~min}$ after birth. There were no significant differences in the gender, age, weight and Apgar score between these two groups ( $\mathrm{p}>0.05)$.

\section{Improved biochemical and oxygenation index}

Compared to control group, serum levels of CK, ALT, AST, creatinine and NSE were significantly lower in treatment group. In addition, $\mathrm{PO}_{2} / \mathrm{FiO}_{2}$ was significantly higher in treatment group $(\mathrm{p}<0.05$, Table I).

\section{Improves neurobehavioral ability}

Compared to control group, neurobehavioral scores were not significantly different in the treatment group on day 1 ( $>0.05)$, but were significantly higher in the treatment group on day 14 and day $28(\mathrm{p}<0.05$, Table II).

\section{Increased the survival of neonates}

At 14 days after treatment, we calculated the survival rate of neonates in two groups. The survival rate was significantly higher in treatment group $(77.5 \%)$ than in control group (55\%) (Table III). The survival curve also showed that the survival rate was better in treatment group than in control group (Figure 1).

\begin{tabular}{|c|c|c|c|c|c|c|c|c|}
\hline \multicolumn{9}{|c|}{ Table I } \\
\hline \multicolumn{9}{|c|}{ Biochemical and oxygenation index in two groups } \\
\hline Group & Day & $\mathrm{n}$ & $\begin{array}{c}\text { CK } \\
(\mathrm{u} / \mathrm{L})\end{array}$ & $\begin{array}{l}\text { ALT } \\
(\mathrm{u} / \mathrm{L})\end{array}$ & $\begin{array}{c}\text { AST } \\
(\mathrm{u} / \mathrm{L})\end{array}$ & $\begin{array}{l}\text { Creatinine } \\
\text { (umol/L) }\end{array}$ & $\begin{array}{c}\text { NSE } \\
(\mathrm{pg} / \mathrm{mL})\end{array}$ & $\mathrm{PO}_{2} / \mathrm{FiO}_{2}$ \\
\hline Treatment & 1 & 40 & $310.5 \pm 122.6$ & $176.5 \pm 45.6$ & $239.5 \pm 78.6$ & $195.5 \pm 35.6$ & $45.8 \pm 16.5$ & $75.5 \pm 12.3$ \\
\hline Treatment & 7 & 34 & $110.7 \pm 30.8$ & $65.5 \pm 17.5$ & $88.5 \pm 25.3$ & $95.8 \pm 12.6$ & $22.8 \pm 5.7$ & $375.7 \pm 30.8$ \\
\hline Treatment & 14 & 31 & $35.5 \pm 9.3$ & $36.5 \pm 12.3$ & $45.5 \pm 10.6$ & $80.5 \pm 17.1$ & $15.8 \pm 4.3$ & $450.7 \pm 80.1$ \\
\hline Control & 1 & 40 & $305.5 \pm 94.6$ & $180.5 \pm 30.5$ & $231.5 \pm 68.7$ & $189.5 \pm 33.1$ & $43.8 \pm 15.7$ & $80.7 \pm 13.9$ \\
\hline Control & 7 & 26 & $180.7 \pm 64.2$ & $110.5 \pm 18.6$ & $130.5 \pm 40.2$ & $135.5 \pm 18.3$ & $37.2 \pm 6.4$ & $259.6 \pm 36.2$ \\
\hline Control & 14 & 22 & $54.5 \pm 19.4$ & $55.5 \pm 17.8$ & $72.5 \pm 15.7$ & $104.5 \pm 38.7$ & $27.8 \pm 5.9$ & $400.7 \pm 60.4$ \\
\hline$P$ & & & 0.024 & 0.035 & 0.01 & 0.027 & 0.034 & 0.045 \\
\hline$F$ & & & 5.47 & 3.28 & 16.58 & 5.10 & 3.45 & 2.89 \\
\hline
\end{tabular}




\begin{tabular}{|lccrrrr|}
\hline \multicolumn{7}{c|}{ Table II } \\
\hline Group & $\mathrm{n}$ & \multicolumn{7}{c|}{ Neurobehavioral scores in two groups } & \\
\hline Treatment & 40 & Day 1 & $\mathrm{n}$ & Day 14 & $\mathrm{n}$ & Day 28 \\
Control & 40 & $21.4 \pm 1.5$ & 31 & $32.4 \pm 2.2$ & 31 & $37.8 \pm 3.1$ \\
$t$ & & $21.8 \pm 1.5$ & 22 & $26.1 \pm 2.4$ & 22 & $33.0 \pm 3.0$ \\
$\mathrm{p}$ & & 1.1 & & 2.9 & 2.7 \\
\hline
\end{tabular}

\section{Discussion}

In this study, injection of shenfu to neonates with asphyxia significantly reduced the serum levels of creatine kinase, alanine aminotransferase, aspartate aminotransferase, creatinine, and neuron-specific enolase. But the oxygenation index was significantly higher in treatment group on day 7 and day 14 after shenfu injection. In addition, neurobehavioral score was significantly higher in treatment group than in control group. Finally, we compared the survival of neonates in treatment group and control group and the results showed that the survival rate of treatment group (77.5\%) was higher than that of control group (55\%) on the 14th day after shenfu injection.

Since serum creatine kinase levels at day 7 and day 14 after birth were significantly lower in treatment group than in control group, we speculated that shenfu injection help protect the heart from reperfusion injury. In addition, serum trnasaminases and creatinine levels were significantly lower in the treatment group than in control group, suggesting that shenfu injection reduces the injury of ischemia-reperfusion to the liver and kidney. Furthermore, oxygenation index PO2/FiO2 was significantly higher in treatment group than in control group, indicating that shenfu injection improves lung function.

\section{Table III}

The survival rate in two groups on day 14

\begin{tabular}{|l|c|c|c|}
\hline Group & $\mathrm{n}$ & Death $(\mathrm{n})$ & Survival (n) \\
\hline Treatment group & 40 & $9(22.5)$ & $31(77.5)$ \\
Control group & 40 & $18(45.0)$ & $22(55.0)$ \\
\hline
\end{tabular}

The brain is highly vulnerable to hypoxia because of high energy requirements compared to low energy reserves (Hossmann, 1999). The brain regions most sensitive to hypoxia-induced injury are the hippocampus, the dorsolateral caudate nucleus and the reticular nucleus of thalamus. Hypoxia leads to coupling disturbances between brain function and blood flow, glutamate-propagated functional disturbances, free radical mediated changes, disturbances of signaling pathways and gene expression patterns and programmed cell death (Hossmann, 1999). Shenfu injection has been shown to attenuate post-resuscitation cerebral ischemia and reperfusion injury by modulating mitochondrial dysfunction of nerve cells (Gu et al., 2014). Our previous study also showed that shenfu injection protected neonatal hypoxic-ischemic brain injury by preventing neuron apoptosis (Yang et al., 2013). There-

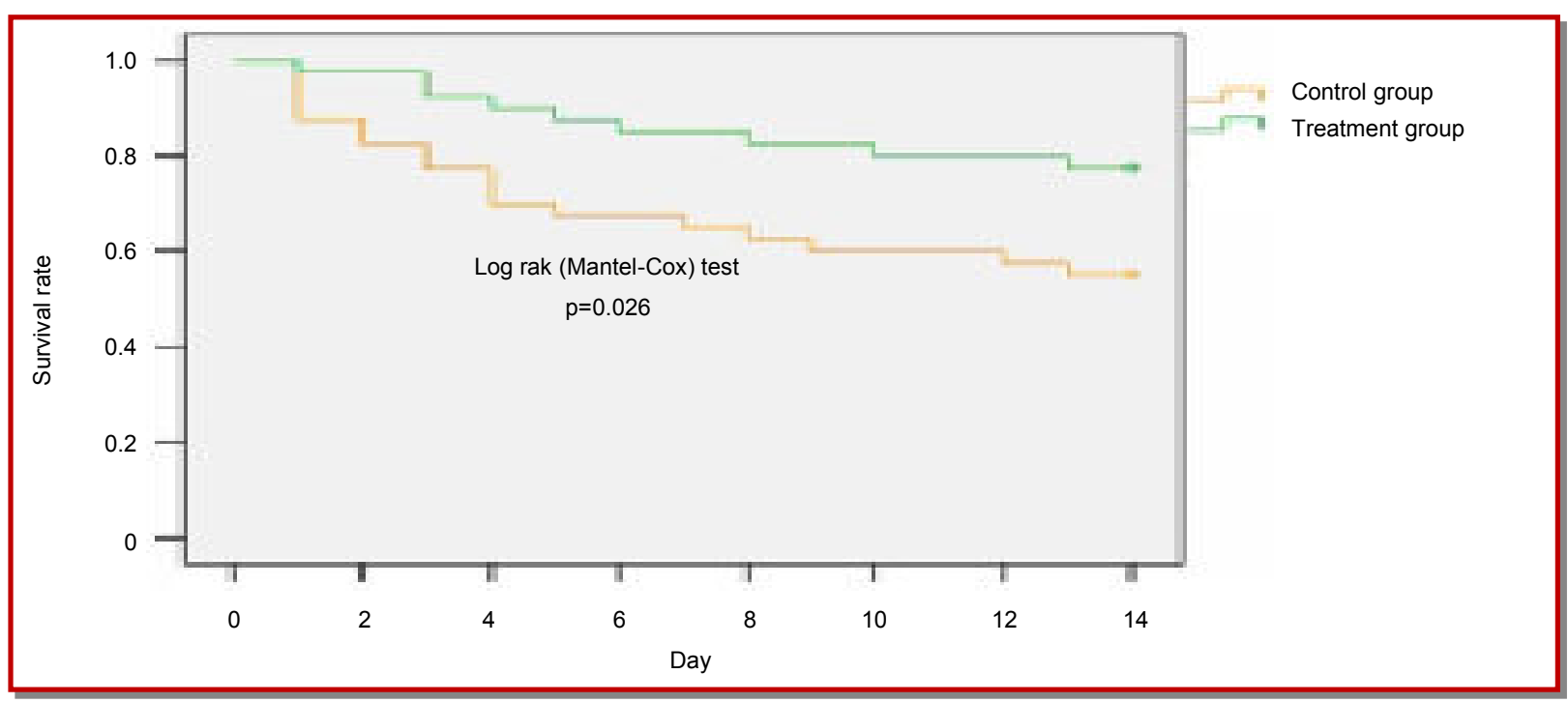

Figure 1: The survival curves of two groups from day 1 to day 14 after birth 
fore, in this study we evaluated the effects of shenfu injection on the brains of neonates after resuscitation procedure. The results showed that serum neuronspecific enolase level was significantly reduced in treatment group compared to control group. Serum level of neuron-specific enolase is proposed as a marker for acute ischemic stroke, because it is higher in stroke patients than in controls and correlated with the volume of infarcted tissue (Anand and Stead, 2005). These data indicate that shenfu injection relieves acute ischemic stroke. Furthermore, we assessed neurobehavioral scores of the neonates and found that neurobehavioral scores were significantly higher after shenfu injection.

The active components of shenfu injection are ginsenosides and aconitine. Pharmacological studies have shown that ginsenosides exhibit protective effects against stress, oxidation, myocardial ischemia. Mechanistically, ginsenosides protected the mitochondria from calcium-induced damage by attenuating mitochondrial swelling, preserving mitochondrial membrane potential and decreasing the production of reactive oxygen species. Moreover, ginsenosides reduced the release of cytochrome $c$ and apoptosisinducing factor and prevented mitochondria-mediated apoptosis following ischemia (Ye et al., 2011). These active components of shenfu injection may mediate the protective effects of shenfu on neonates after severe asphyxia resuscitation.

\section{Conclusion}

Shenfu injection could protect the function of the brain, heart, lung, liver and kidney by attenuating ischemia reperfusion after severe asphyxia resuscitation, improve neurobehavioral ability and increase the survival of neonates.

\section{Conflict of Interest}

The authors declare that they have no competing interest.

\section{References}

Anand N, Stead LG. Neuron-specific enolase as a marker for acute ischemic stroke: A systematic review. Cerebrovasc Dis. 2005; 20: 213-19.
Bao XL, Yu RJ, Li ZS, Zhang BL. Twenty-item behavioral neurological assessment for normal newborns in 12 cities of China. Chin Med J (Engl). 1991; 104: 742-46.

Goel N, Gupta B. Maternal risk factors affecting perinatal mortality. Indian Pediatr. 2012; 49: 188-89.

Gu W, Hou X, Zhou H, Li C. Protective effect of shenfu injection on neuronal mitochondrial function in a porcine model of prolonged cardiac arrest. Evid Based Complement Alternat Med. 2014; 2014: 523847.

Guo ZJ, Li CS. Therapeutic effects of shenfu injection on post cardiac arrest syndrome. Chin J Integr Med. 2013; 19: 71620 .

Hossmann KA. The hypoxic brain: Insights from ischemia research. Adv Exp Med Biol. 1999; 474: 155-69.

Hou X, Li C, Gu W, Guo Z, Yin W, Zhang D. Effect of shenfu on inflammatory cytokine release and brain edema after prolonged cardiac arrest in the swine. Am J Emerg Med. 2013; 31: 1159-64.

Ji XF, Ji HB, Sang DY, Wang S, Yang L, Li CS. Shenfu injection reduces impaired myocardial $\beta$-adrenergic receptor signaling after cardiopulmonary resuscitation. Chin Med J (Engl). 2013; 126: 697-702.

Leone TA, Finer NN. Shock: A common consequence of neonatal asphyxia. J Pediatr. 2011; 158 (2 Suppl): e9-12.

Wang J, Qiao LF, Yang GT. Role of shenfu injection in rats with systemic inflammatory response syndrome. Chin J Integr Med. 2008; 14: 51-55.

Yang LJ, Wang J, Tian ZF, Yuan YF. Shenfu injection attenuates neonatal hypoxic-ischemic brain damage in rat. Neurol Sci. 2013; 34: 1571-74.

Ye R, Zhang X, Kong X, Han J, Yang Q, Zhang Y, Chen Y, Li $\mathrm{P}$, Liu J, Shi M, Xiong L, Zhao G. Ginsenoside Rd attenuates mitochondrial dysfunction and sequential apoptosis after transient focal ischemia. Neuroscience 2011; 178: 169-80.

Yin W, Guo Z, Li C. Comparison of epinephrine and shenfu injection on resuscitation outcomes in a porcine model of prolonged cardiac arrest. Chin Med J (Engl). 2014; 127: 72428.

Zhang MY, Ji XF, Wang S, Li CS. Shenfu injection attenuates post-resuscitation lung injury in a porcine model of cardiac arrest. Resuscitation 2012; 83: 1152-58.

Zheng SD, Wu HJ, Yu SP, Ren JX, Duo WW, Ma ZC, Gao Y, Wang SQ, Liu YN. Shenfu injection suppresses inflammation by targeting haptoglobin and pentraxin 3 in rats with chronic ischemic heart failure. Chin J Integr Med. 2015; 21: 22 -28 . 


\section{Your feedback about this paper}

1. Number of times you have read this paper 0

2. Quality of paper Click

3. Your comments

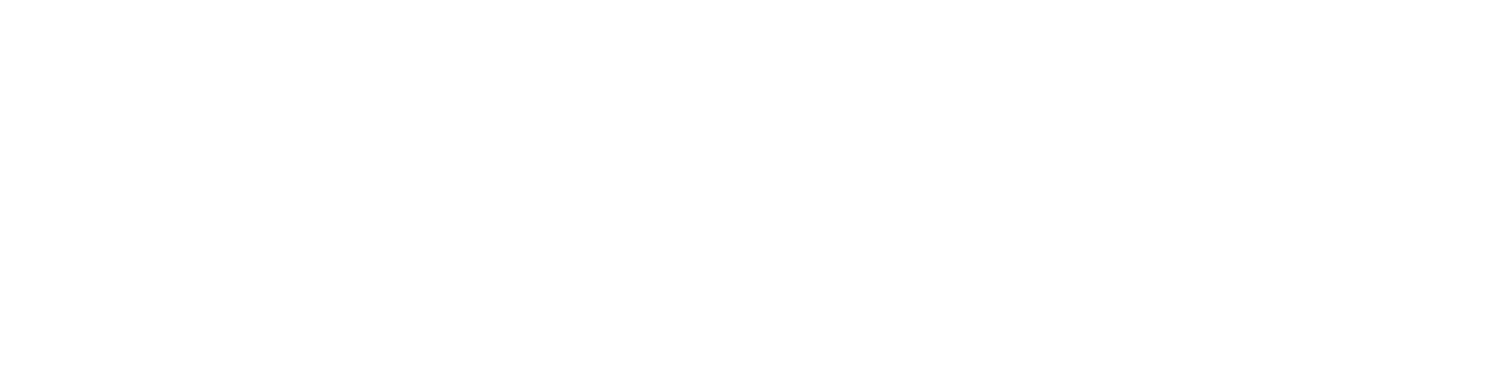

